\title{
Collagen/Beta-Tricalcium Phosphate Based Synthetic Bone Grafts via Dehydrothermal Processing
}

\author{
Burcu Sarikaya ${ }^{1,2}$ and Halil Murat Aydin ${ }^{3,4}$ \\ ${ }^{1}$ Institute of Science, Bioengineering Division, Hacettepe University, 06800 Ankara, Turkey \\ ${ }^{2}$ Faculty of Engineering and Architecture, Genetics and Bioengineering Department, Kastamonu University, 37150 Kastamonu, Turkey \\ ${ }^{3}$ BMT Calsis Health Technologies Co., 06980 Ankara, Turkey \\ ${ }^{4}$ Environmental Engineering Department \& Bioengineering Division and Centre for Bioengineering, Hacettepe University, \\ 06800 Ankara, Turkey
}

Correspondence should be addressed to Halil Murat Aydin; hmaydin@hacettepe.edu.tr

Received 28 April 2015; Revised 10 August 2015; Accepted 13 August 2015

Academic Editor: Giuseppe Cama

Copyright (C) 2015 B. Sarikaya and H. M. Aydin. This is an open access article distributed under the Creative Commons Attribution License, which permits unrestricted use, distribution, and reproduction in any medium, provided the original work is properly cited.

\begin{abstract}
Millions of patients worldwide remain inadequately treated for bone defects related to factors such as disease or trauma. The drawbacks of metallic implant and autograft/allograft use have steered therapeutic approaches towards tissue engineering solutions involving tissue regeneration scaffolds. This study proposes a composite scaffold with properties tailored to address the macro- and microenvironmental conditions deemed necessary for successful regeneration of bone in defect areas. The biodegradable scaffold composed of porous beta-tricalcium phosphate particles and collagen type I fibers is prepared from a mixture of collagen type-I and $\beta$-tricalcium phosphate $(\beta$-TCP) particles via lyophilization, followed by dehydrothermal (DHT) processing. The effects of both sterilization via gamma radiation and the use of DHT processing to achieve cross-linking were investigated. The impact of the chosen fabrication methods on scaffold microstructure and $\beta$-TCP particle-collagen fiber combinations were analyzed using X-ray diffractometry (XRD), scanning electron microscopy (SEM), Fourier transform infrared spectroscopy (FTIR), differential scanning calorimetry (DSC), and microcomputerized tomography ( $\mu$-CT). Electron spinning resonance (ESR) analysis was used to investigate free radicals formation following sterilization. Results revealed that the highly porous (65\% porosity at an average of $100 \mu \mathrm{m}$ pore size), mechanically adequate, and biocompatible scaffolds can be utilized for bone defect repairs.
\end{abstract}

\section{Introduction}

Accidents, fractures, osteoporosis, tumor removal, and hereditary diseases where large bone defects may occur necessitate appropriate methods for the regeneration of lost tissues [1]. Many clinical approaches to bone repair and regeneration have been reported. Autografts use, the current gold standard for treating critical-sized bone defects, is limited by factors such as donor site morbidity, the risk of bone resorption, and poor availability and there is thus growing interest in bone graft substitutes $[2,3]$. The dry weight of the natural bone matrix consists of $75 \%$ inorganic material, calcium phosphate, and around $22 \%$ organic material, collagen along with low amounts of proteoglycans, peptides, and lipids [4]. The extracellular matrix, which is composed of a network of collagen fibrils offering an environment for cell proliferation and migration, can be decellularized. However, the risk of pathogen or disease transfer has led, instead, to the development of scaffolds that can mimic the ECM in in vitro conditions [5]. Scaffolds that can be used for bone regeneration are fascinating as they have the ability to be produced with desirable properties, can be sterilized to display low immunogenicity, shaped to the defect site, optimized, and altered accordingly, and can be used in combination with cells and growth factors [1]. Materials used in bone grafts must allow for osteoblasts to interact with other osteoblasts, proliferate, and migrate [6].

Materials used for scaffolds must be biocompatible, biodegradable, mechanically strong, and osteointegrative. Ceramic materials are commonly used in clinical applications; 
hydroxyapatite (HA) is one of the most widely used ceramics in medicine. Even though HA displays high mechanical strength, it does not possess interconnected porosity, a feature essential to the vascularization of newly formed tissue. Furthermore, as HA is nonbiodegradable it cannot be replaced with new bone tissue over time [7]. To avoid such limitations we have preferred the incorporation of highly pure and porous $\beta$-TCP particles (SupraBone (BMT Calsis Co., Turkey)) in the scaffolds developed in our study. $\beta$-TCP has a $\mathrm{Ca} / \mathrm{P}$ ratio very similar to that of natural bone tissue, is biodegradable, and can be produced to exhibit ideal pore sizes [8]. However, this material cannot be used alone in the regeneration of bone due to its brittleness. For this reason it is more effective to use TCP in conjunction with the primary components of bone such as collagen. The use of collagen-only scaffolds is also not desirable due to its early degradation within the body and low resistance [9]. Furthermore, there are difficulties associated with the combination of collagen with other biopolymers as internal shear stresses between the materials tend to compromise the mechanical integrity of the scaffold [8]. Hybrid scaffolds that unite the elasticity of collagen and the high mechanical resistance of $\beta$-TCP offer viable solutions to the current problems encountered in hard tissue engineering $[6,10]$.

Many methods can be employed to create pore of desired size, density, and interconnectivity in tissue engineering scaffolds [11]. Chemical approaches, where the removal of solvents involves arduous processing, carry a risk of causing tissue damage if residue remains within the material [12]; we have thus utilized a lyophilization technique in our study to achieve porosity. As the $\beta$-TCP and collagen composite scaffolds are likely to degrade/disintegrate before new tissue is formed, they need to be cross-linked for in vivo use. Agents such as diphenylphosphoryl azide (DPPA) and glutaraldehyde (GTA) have previously been used to cross-link collagen [13]. Even though the cross-linking agents are able to give fast and effective results, the complete removal of solvents used in the process is not always successful. To achieve cross-linking whilst avoiding the possibility of such adverse effects, a nonchemical method, namely, dehydrothermal (DHT) processing, can be used. As strong links between the ceramic phase and organic phase of the collagen/ $\beta$-TCP scaffolds can be formed via DHT processing, degradation time is increased, cell proliferation is increased, and controlled release of growth factors becomes possible [3].

In this work, porous collagen/ $\beta$-TCP composites were prepared by acid-treating the collagen, dispersing fine $\beta$-TCP particles throughout the mixture, and lyophilizing in order to obtain desired uniform microenvironments within the composites. To minimize early degradation of the scaffold in the body, DHT processing to cross-link the collagen/ $\beta$-TCP scaffold was used. The effects of cross-linking and sterilization parameters on $\beta$-TCP particles and collagen properties were investigated.

\section{Materials and Methods}

2.1. Preparation of Porous $\beta$-TCP/Collagen Scaffolds. Collagen type-I (RUP, Czech Republic) was dispersed in a diluted acetic acid solution $(0.1 \mathrm{~N})$ at room temperature. To completely dissolve collagen, the mixture was stirred for 5 minutes using an overhead blender at the speed of $500 \mathrm{rpm}$. In order to deliver the maximum amount of ceramic particles without compromising the handling and wettability properties of the final graft material; an $80: 20 \mathrm{w} / \mathrm{w}$ ratio was selected for $\beta$ TCP/collagen mixture. A designed amount of $\beta$-TCP powders was slowly added into the collagen suspension by stirring. $\beta$-TCP (SupraBone, $0,5-1 \mathrm{~mm}$ particle size) was kindly donated by BMT Calsis Health Technologies, Co., Turkey. This particle size was found suitable since bigger ceramic particles could lead to lower packing density and fragile structure. After the addition of $\beta$-TCP, the slurry mixture was stirred for 3 minutes at a speed of $250 \mathrm{rpm}$. The slurry was casted into a Teflon mold (dimensions $5 \mathrm{~mm} \times 25 \mathrm{~mm} \times$ $100 \mathrm{~mm}$ ) and stored at $-80^{\circ} \mathrm{C}$ for 3 hours [14]. Subsequently, the frozen mixture was lyophilized to obtain a porous collagen/ $\beta$-TCP composite. After freeze-drying the porous composites, the matrices were placed into the vacuum oven for dehydrothermal processing at $3 \mathrm{mbar}$ and $110^{\circ} \mathrm{C}$ operating conditions for 1,3 , and 5 days. It should be noted that the shorter treatment times may lead to incomplete cross-linking while longer periods could effect the chemical structure. Some of the bone grafts were sterilized by using gamma irradiation with an average prescribed dose of $25 \mathrm{kGy}$ at room temperature, whilst a control batch remained unsterilized for comparative analysis purposes.

2.2. Characterization of Scaffolds. The crystalline phases of the porous composites were determined with an X-ray diffractometer (XRD) using the RIGAKU, RINT 2000 system. The surface morphology of $\beta$-TCP/collagen scaffolds was examined using a scanning electron microscope (SUPRA 50VP, Germany). The scaffolds were sputter-coated with gold-palladium alloy before SEM imaging. The operated voltage was set at $20 \mathrm{kV}$. FTIR analysis was carried out to determine components of $\beta$-TCP/collagen scaffolds and characterize the interactions between $\beta$-TCP particles and collagen fibrils using an attenuated total reflection system on a (BRUKER TENSOR 27, USA) spectrometer. Further analysis involving Fourier transform infrared (FTIR) spectroscopy was adopted using the $\mathrm{KBr}$ pellet method and FTIR spectra were recorded at $4.0 \mathrm{~cm}^{-1}$ resolution. A dry system was used to prevent atmospheric moisture. Differential thermal analyses (DTA) were performed for the determination of thermal transitions. The thermal behaviors of the $\beta$-TCP/collagen scaffolds were also investigated by thermogravimetric analysis (TGA), and differential thermal analysis (DTA) using the NETZSCH thermal analyzer (STA 449 F3 JUPITER, Germany). For TG-DTA measurement, the heating rate was set at $10^{\circ} \mathrm{C} / \mathrm{min}$ under flowing air. An X-band Electron Spin Resonance (ESR) spectrometer (Bruker ELEXSYS E580, USA), fitted with a $\mathrm{TE}_{102}$ cavity and operating at $9.85 \mathrm{GHz}$ microwave frequency and $100 \mathrm{kHz}$ magnetic-field modulation frequency, was used to test all samples in this study.

In order to reveal mechanical properties, three-point bending strength tests were carried out using a Zwick/Roell Z250 mechanical tester. The size of the specimens was $\sim 5 \mathrm{~mm}$ $\times 12,5 \mathrm{~mm} \times 50 \mathrm{~mm}$. The $0,2 \mathrm{~N}$ load was applied over a $10 \mathrm{~mm}$ 


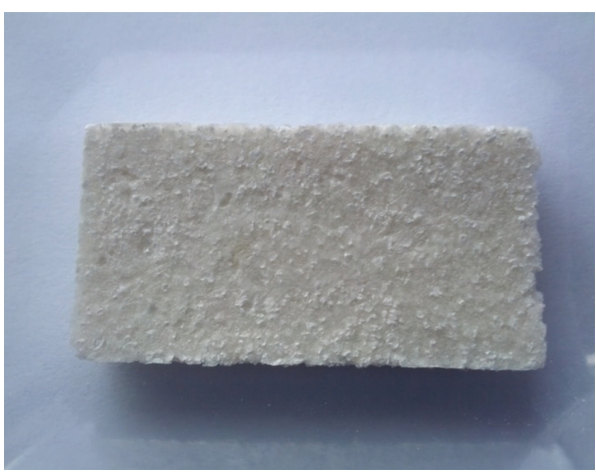

(a)

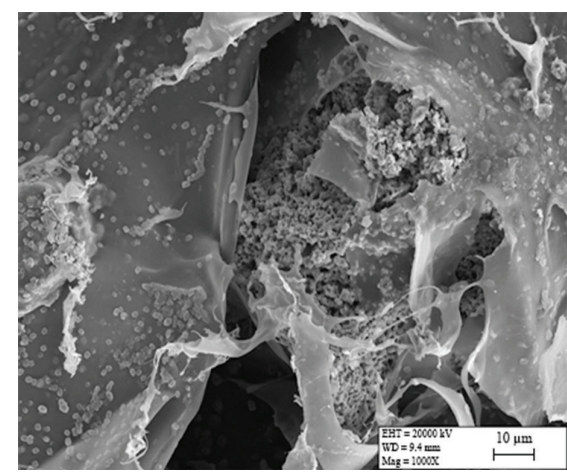

(b)

FIGURE 1: Characteristics of the collagen/ $\beta$-TCP scaffolds. (a) Macroscopic image, (b) SEM image of gamma irradiation sterilized scaffold $(\times 1000)$.

span and at the midpoint of the $12,5 \mathrm{~mm} \times 50 \mathrm{~mm}$ surface. All tests were performed using a crosshead speed of $2 \mathrm{~mm} / \mathrm{min}$. The compression strength of porous scaffolds was also measured using a Zwick/Roell Z010 mechanical tester at a crosshead speed of $2 \mathrm{~mm} / \mathrm{min}$. The samples were rectangular in shape, with dimensions $5 \mathrm{~mm}$ in height and $10 \mathrm{~mm} \times$ $10 \mathrm{~mm}$ in cross section. During compression test, the $0,25 \mathrm{~N}$ load was applied until densification of the porous samples started to occur.

A micro-CT system (SkyScan 1172, USA) was used to quantify the 3D microstructural properties of the collagen/ $\beta$ TCP scaffolds. Isotropic slice data were obtained by the system and reconstructed into two-dimensional images and analyzed to produce 3D images for quantitative architectural parameters. The data were further analyzed by the software CT Analyzer (SkyScan, USA). Porosity, pore sizes, and pore distribution were measured from the constructed 3D model.

To evaluate the degree of water uptake, the cross-linked scaffold samples with the specific dimensions $(10 \mathrm{~mm} \times$ $12 \mathrm{~mm} \times 5 \mathrm{~mm})$ were accurately weighed $\left(W_{0}\right)$ and immersed in $3 \mathrm{~mL}$ PBS solution at $37^{\circ} \mathrm{C}$ according to [15]. The samples were reweighed at regular time intervals $\left(W_{S}\right)$ to find the equilibrium swelling. The water uptake was defined as $\left(W_{S}-\right.$ $\left.W_{0}\right) \times 100 / W_{S}$.

2.3. Cell Culture Assays. The scaffolds consisting of collagen and $\beta$-tricalcium phosphate (TCP) were cut to size of $24 \mathrm{~mm}$ $\times 47 \mathrm{~mm}$ prior to the commencement of the experiment. To assess the possible cytotoxicity of the scaffolds, they were seeded with a commonly used osteosarcoma cell line, MG63. The cells were exclusively cultured for this experiment using a specifically supplemented medium consisting of $\alpha$-MEM, $10 \%$ FBS, $1 \%$ L-glutamine, $1 \%$ antibiotics, and antimycotic. Prior to seeding, scaffolds were cut to a specific size $(6 \mathrm{~mm} \times$ $6 \mathrm{~mm}$ ) and then soaked in fully supplemented MG63 medium for $30 \mathrm{~min}$. To assess initial seeding efficiency, the scaffolds were then seeded in a sterile Petri dish. $0.5 \times 10^{6}$ cells were seeded in each scaffold in a volume of $100 \mu \mathrm{L}$ supplemented MG63 medium. The cell suspension was seeded with 5 separate volumes of $20 \mu \mathrm{L}$ to ensure a homogenous distribution within the scaffold. It was also hoped that this method of seeding would prevent cell suspension leaking from the scaffolds until cell attachment was achieved. The scaffolds were then incubated $\left(37^{\circ} \mathrm{C}\right.$ at $\left.5 \% \mathrm{CO}_{2}\right)$ for $2 \mathrm{hrs}$ before being transferred to individual wells of a 24 -well plate. Once transferred, the wells were topped up with $1 \mathrm{~mL}$ supplemented MG63 medium. After $24 \mathrm{hrs}$, half the wells were topped up with an additional $1 \mathrm{~mL}$ supplemented MG63 medium (bringing the volume of half the wells to $2 \mathrm{~mL}$ ). This was intended to assess if medium volume affected cytotoxicity and scaffold integrity. The scaffolds were imaged at $2 \mathrm{hrs}, 24 \mathrm{hrs}, 48 \mathrm{hrs}, 72 \mathrm{hrs}$, and 192 hrs. The $\mathrm{pH}$ of the supplemented MG63 medium was tested prior to seeding and again after the experiment was complete.

For the disintegration experiment, eight scaffolds (cut to the same dimensions as those used in the seeding experiment) were used to assess and monitor the levels of disintegration within a number of mediums: DMEM, $\alpha$ MEM, supplemented MG63 media, and PBS. The scaffolds were cut and immediately transferred to individual wells of a 24-well plate. Each well was then topped up with their designated medium (3x scaffolds were each soaked in DMEM, $\alpha$ MEM, supplemented MG63 media, and PBS). The scaffolds were imaged every $30 \mathrm{~min}$ for the first $5 \mathrm{hrs}$ and twice again at $24 \mathrm{hrs}$. Immediately following the initial imaging at $24 \mathrm{hrs}$, an attempt was made to transfer the scaffolds to new wells with images being taken again immediately after. This was intended to evaluate whether the moving of the scaffolds after $2 \mathrm{hrs}$ of postseeding could have affected the integrity of the scaffolds. MTT analysis was carried out to evaluate cell viability at $45 \mathrm{hrs}$ and $70 \mathrm{hrs}(n=4 \times 3)$ using manufacturers protocols. A standard curve was made using the same methods as those previously mentioned with cell numbers of $0.2 \times 10^{6}$ to $1 \times 10^{6}$.

\section{Results}

3.1. Scaffold Characterization. Figure 1 shows the morphology and structure of collagen/ $\beta$-TCP scaffolds. The composite scaffolds were white and opaque with a spongy appearance (Figure 1(a)) and exhibited an interconnected porous structure (Figure 1(b)). $\beta$-TCP particles were distributed well 
TABLE 1: Thermal properties of collagen/ $\beta$-TCP scaffold.

\begin{tabular}{lccc}
\hline Groups & Total weight loss $(\%)$ & ${ }^{*} T_{d}\left({ }^{\circ} \mathrm{C}\right)$ & ${ }^{* *} T_{m}\left({ }^{\circ} \mathrm{C}\right)$ \\
\hline Sterile sample & 33,3 & 133,3 & 46,6 \\
Nonsterile sample & 28,6 & 120,0 & 53,3 \\
\hline
\end{tabular}

${ }^{*}$ Temperature at degradation. ${ }^{* *}$ Temperature at denaturation.

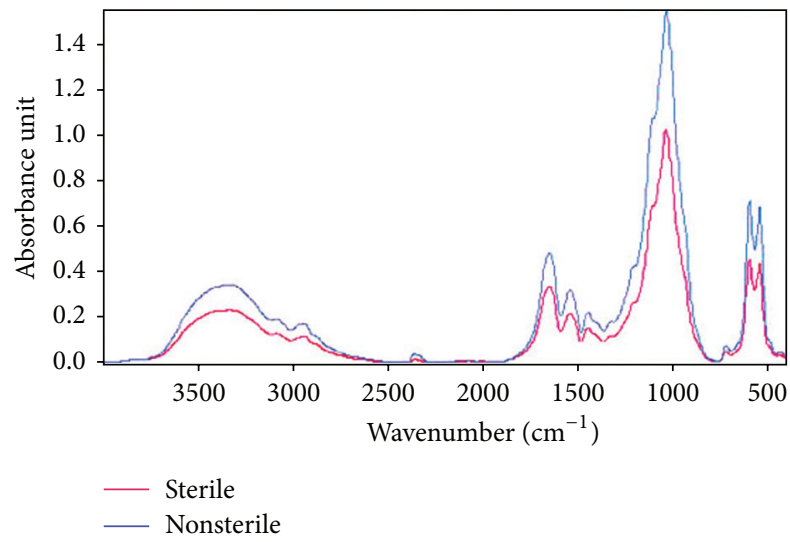

FIGURE 2: FTIR spectra of the sterile and nonsterile collagen/ $\beta$-TCP scaffolds.

and homogeneously in the skeleton network of collagen fibrils. The interconnected porous scaffold structures have beneficial effects on cell proliferation, migration, and nutritive transportation in bone tissue engineering. It was also noted that the organic and inorganic phases were combined appropriately following gamma sterilization.

In the FTIR spectra (Figure 2), adsorption bands were clearly detected, which corresponded to $\beta$-TCP $\left(\mathrm{PO}_{4}{ }^{3-}: 1000\right.$, 600 , and $\left.580 \mathrm{~cm}^{-1}\right)$ and collagen $\left(\mathrm{COO}^{-}: 1630,1543\right.$, and $1452 \mathrm{~cm}^{-1}$ ), respectively. The FTIR data shows the collagen and ceramic phases have not undergone significant change following poststerilization.

XRD investigations detected a peak profile of collagen $/ \beta$ TCP scaffold overlapped with the product of $\beta$-TCP powder. Mineralogical analysis results examined in sterile and nonsterile samples were found to contain $\left[\mathrm{Ca}_{3}\left(\mathrm{PO}_{4}\right)_{2}\right]$ phase. Collagen type-I did not peak in XRD analysis due to its amorphous structure but peak between 25 and $35^{\circ}$ in sterile and nonsterile samples revealed the presence of amorphous structure. There are not any differences observed between sterile and nonsterile samples peak when graphics overlapped (Figure 3).

Following thermal analyses, a three-stage degradation was observed in TG-DTA analysis that determines the weight loss. The first endothermic peak in thermogram that related with weight loss indicates evaporation of water. Second exothermic peak in the range of $200-400^{\circ} \mathrm{C}$ implies collagen degradation. A noticeable difference between the DTA results of sterile and nonsterile samples was not seen. Sterile and nonsterile samples exhibit similar thermal behavior (Table 1).

There is no ESR signal obtained from irradiated scaffolds. In the absence of fluctuation in 3500-Gauss magnetic field of

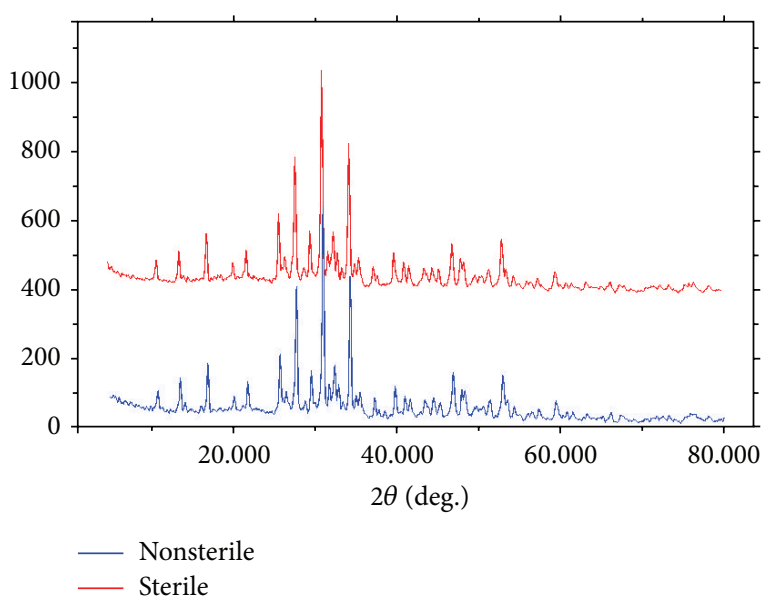

FIGURE 3: XRD patterns of collagen/ $\beta$-TCP scaffold.

TABLE 2: Compression modulus of scaffolds at 1-, 3-, and 5-day DHT processing time $(n=3)$.

\begin{tabular}{lccc}
\hline Treatment time & 1 day & 3 days & 5 days \\
\hline Sterile $(\mathrm{MPa})$ & $2,32 \pm 0,11$ & $3,07 \pm 0,15$ & $2,22 \pm 0,11$ \\
Nonsterile $(\mathrm{MPa})$ & $3,67 \pm 0,18$ & $3,45 \pm 0,17$ & $2,19 \pm 0,1$ \\
\hline
\end{tabular}

TABLE 3: Scaffold parameters assessed by micro-CT analysis.

\begin{tabular}{lc}
\hline Parameter & Amount \\
\hline Total porosity $(\%)$ & 65,63 \\
Open porosity $(\%)$ & 65,54 \\
Surface density $(1 / \mathrm{mm})$ & 8,84 \\
Pixel size $(\mu \mathrm{m})$ & 13,72 \\
\hline
\end{tabular}

free radical formation, we concluded that free radical formation was not observed in scaffolds after gamma sterilization at $25 \mathrm{kGy}$ dose irradiation.

In order to see the effects of DHT processing times on the mechanical properties, compression tests were performed. The summary of the mechanical properties is given in Table 2. It was noted that a DHT processing time of 3 days is optimal, since after this point the compression modulus of the samples showed declination. In addition to the compression tests, a three-point bending test was also performed. According to three-point bending tests, Young's modulus and strength values for the sterile samples were found as $14.12 \pm 0,7 \mathrm{MPa}$ and $0.77 \pm 0,03 \mathrm{MPa}(n=3)$, respectively.

Detailed analysis results of micro-CT images are given in Table 3. The representative micro-CT images of the scaffolds were shown in Figure 4. Scaffolds have an open and porous architecture with porosity of around $65,63 \%$ in the dry stage. It was found that the ceramic phase integrated into the matrix had a micro- and nanopore structure and this had an effect on these results.

The results of the water uptake experiment performed for the cross-linked scaffolds revealed that all the scaffolds reached the equilibrium swelling point in less than 48 hours. As observed in Figure 5, water content of the scaffolds was 


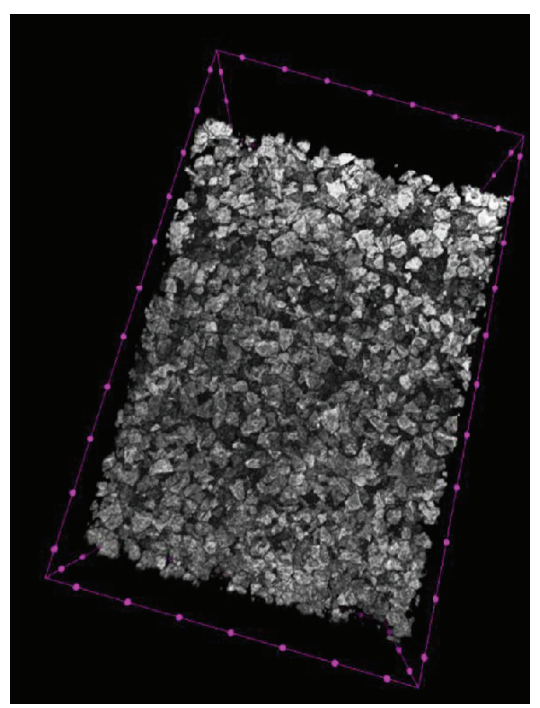

(a)

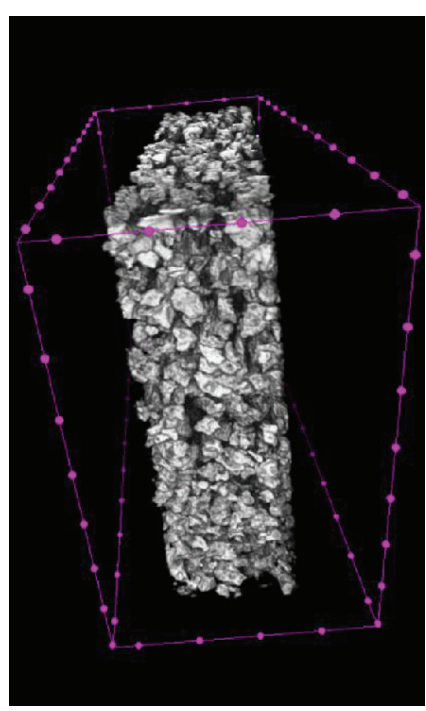

(b)

FIgURE 4: Micro-CT images of a $\beta$-TCP/collagen scaffold. (a) Top-view of scaffold. (b) Side-view of scaffold.

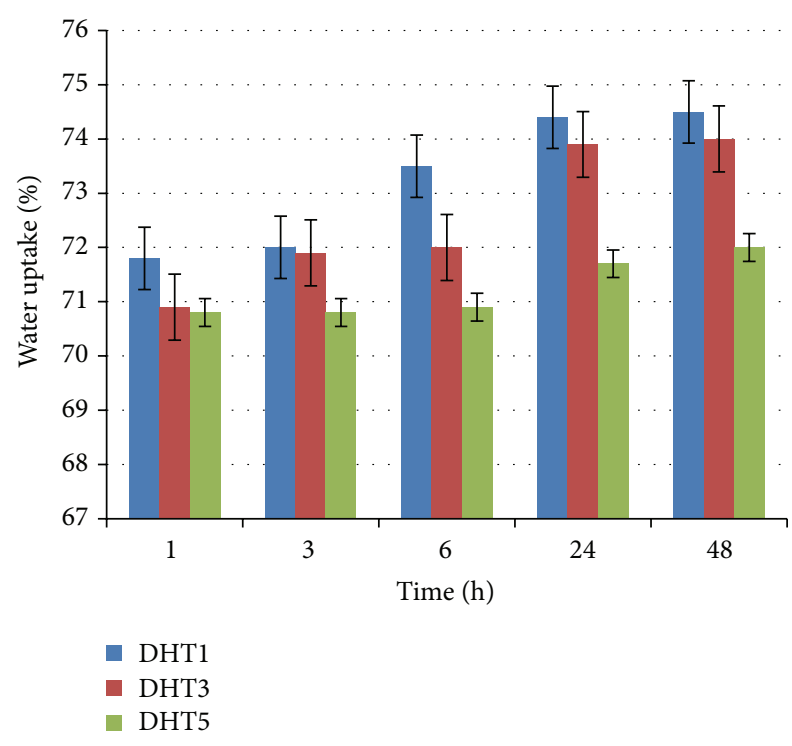

FIGURE 5: Water uptake of the $\beta$-TCP/collagen scaffolds after 48 hours. Error bars show means \pm standard deviation for $n=3$.

inversely proportional to the DHT processing time (DHT1, DHT3, and DHT5 denote 1,3 , and 5 days of DHT treatment).

3.2. In Vitro Disintegration Tests. Disintegration was evident in all four mediums within just $1 \mathrm{hr}$ of being immersed. Within 5 hours, the disintegration had reached a point where the scaffolds had begun to disintegrate (Figure 6). After $24 \mathrm{hrs}$, the scaffolds had become so disintegrated that they could not be transferred to another well. The scaffolds had become too soft and could not be handled.

The initial seeding of the scaffolds was considered successful given that each scaffold accepted the supplemented
MG63 medium (30 min soaking) and cellular solution without leaking. It was noted when soaking and seeding the scaffolds that when more time was given to the scaffolds to accept a solution, they retained the solution better (Figure 7).

After 2 hours incubation $\left(37^{\circ} \mathrm{C}\right.$ at $\left.5 \% \mathrm{CO}_{2}\right)$, however, all of the scaffolds had begun to leak and significant volumes of solution containing both cellular debris and scaffold debris were clearly visible escaping from the scaffolds. The scaffolds were transferred to 24-well plates and images taken of the leaked solution. Significant segments of scaffold can clearly be seen in Figure 8 with small clumps of cells remaining unattached from the dish surface.

After $24 \mathrm{hrs}$, it was difficult to distinguish cellular debris from the mass of scaffold debris that had departed from the main scaffold body but nevertheless some cell attachment remained present (Figure 8).

Disintegration of the scaffolds continued $48 \mathrm{hrs}$ after seeding. Within $192 \mathrm{hrs}$ ( 8 days) all of the scaffolds had completely degraded and broken down into small fragments. These fragments are thought to be the TCP component of the scaffolds. Live/Dead staining was carried out on scaffold segments $192 \mathrm{hrs}$ after seeding (Figure 9) and several segments of scaffold showed high cell viability. Fragments were taken from several scaffolds that had completely disintegrated/ degraded with each showing a higher than expected cell viability.

During The MTT evaluation, a standard curve producing an $R^{2}$ value of 0.99 with the lowest cell number used for the curve was $0.2 \times 10^{6}$, just $40 \%$ of the original seeding density. Within just $45 \mathrm{hrs}$, cell viability had dropped below this value and continued to drop up to $70 \mathrm{hrs}$.

\section{Discussions}

In this study, a flexible and biocompatible composite scaffold of collagen and $\beta$-tricalcium phosphate was prepared without 


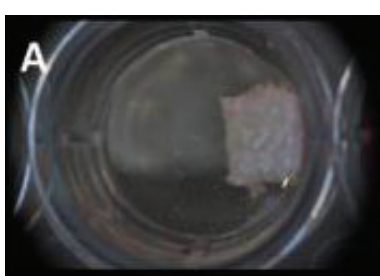

(a)

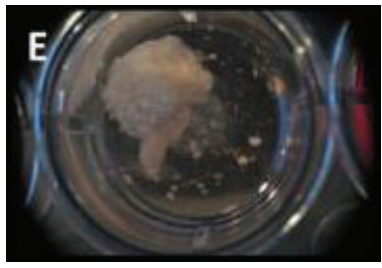

(e)

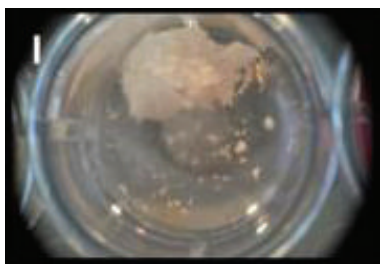

(i)

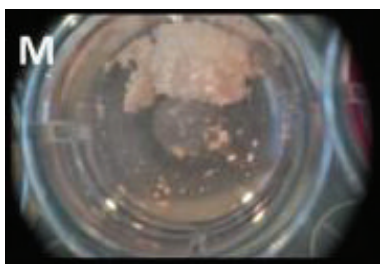

(m)

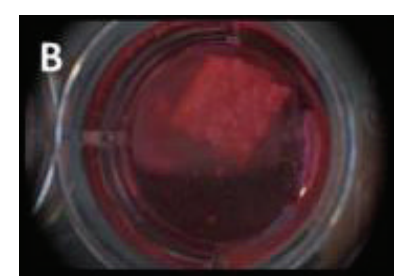

(b)

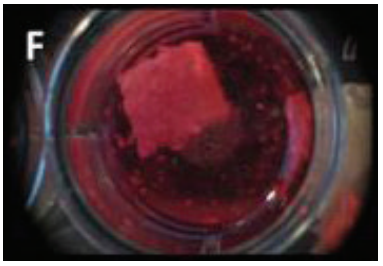

(f)

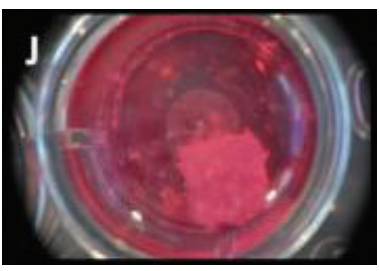

(j)

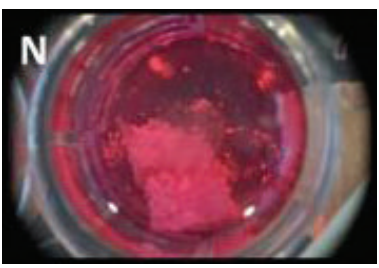

(n)

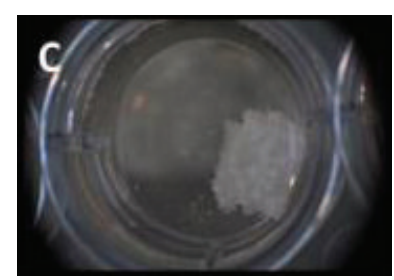

(c)

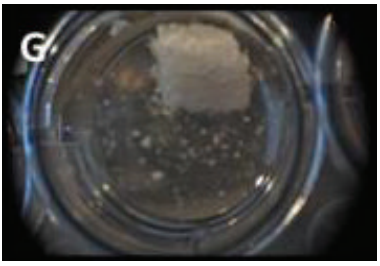

(g)

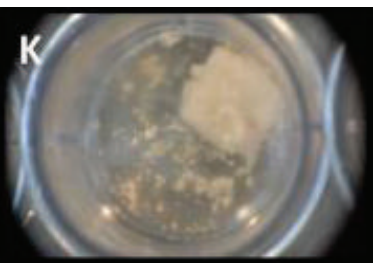

(k)

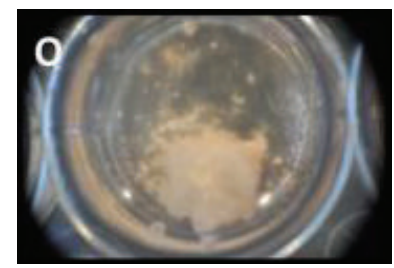

(o)

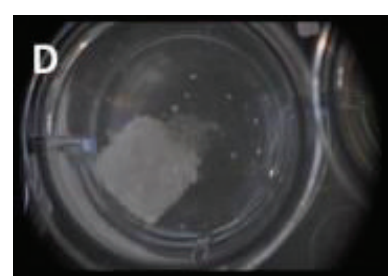

(d)

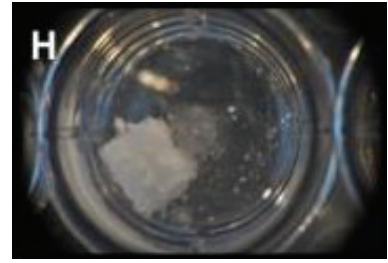

(h)

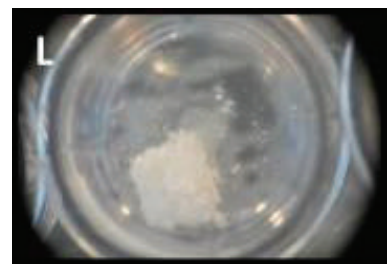

(1)

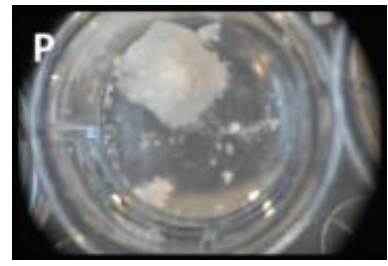

(p)

FiguRE 6: Morphology of scaffolds soaked in four different mediums. (a)-(d) $1 \mathrm{hr}$ after soak. (e)-(h) 5 hrs after soak. (i)-(l) 24 hours after soak. (m)-(p) After transfer (24 hours). (a), (e), (i), (m) Alpha-MEM. (b), (f), (j), (n) DMEM. (c), (g), (k), (o) MG63 supplemented medium. (d), (h), (l), (p) PBS.

using a chemical cross-linker (via dehydrothermal processing, DHT) and the effects of the process on the mechanical, thermal, and biocompatibility properties of collagen $/ \beta$-TCP scaffolds were examined. For this purpose, a mixture of ceramic and collagen phases was prepared and subjected to freeze-drying, resulting in cross-linked scaffolds.

The duration of DHT processing was found to have a critical influence on the mechanical features of the scaffolds prepared. The results from compression testing revealed that a 3day processing time resulted in scaffolds with higher mechanical strength. Increasing the time of exposure to 5 days was found to increase the amount of collagen denaturation in the scaffolds. Although the increase of DHT temperature improves cross-link density, the increase in DHT processing time was found to be inversely proportional to cross-link density, coherent with reported literature [16]. However, the compressive modulus of the scaffolds, determined to be between 2.2 and 3.0 MPa, remains significantly higher than those of similar scaffolds reported in literature [16-19].
FTIR analysis results have established that the collagen fibers were cross-linked via DHT. Additionally, the FTIR spectrum indicates that gamma irradiation further increased cross-link density. Bands up to a width of $1200 \mathrm{~cm}^{-1}$ belong the $\mathrm{PO}_{4}{ }^{-3}$ of $\beta$-TCP [20]. Although the amide-I $\left(1639 \mathrm{~cm}^{-1}\right)$, amide-II $\left(1543 \mathrm{~cm}^{-1}\right)$, and amide-III $\left(1452 \mathrm{~cm}^{-1}\right)$ bands of collagen are quite similar to those of unmodified collagen, the increase in cross-link density due to the interaction between carboxyl and amino groups has led to a shift in the wavenumber and decrease in the area below the peak [21]. Additionally, a cross-link between lysine and alanine may have occurred due to DHT processing; however, this may remain hidden in the FTIR spectra [16].

The denaturation temperature of collagen was found, with differential scanning calorimetry analysis, to be about $50^{\circ} \mathrm{C}$. Whilst the denaturation temperature $\left(T_{d}\right)$ causes the destruction of the triple-helix structure of collagen, the degradation temperature $\left(T_{m}\right)$ relates to the breaking of covalent bonds within the collagen molecule [22]. Several factors including 


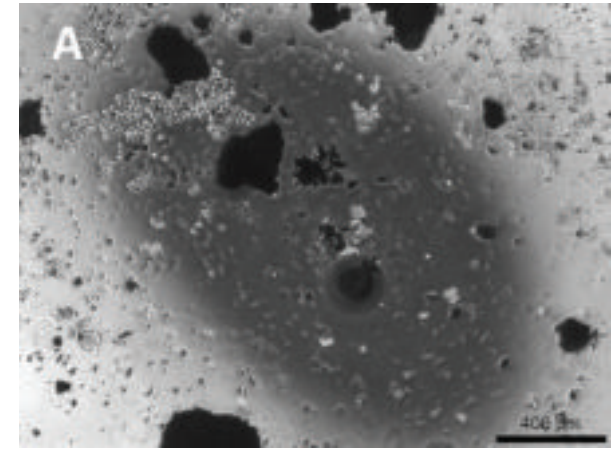

(a)

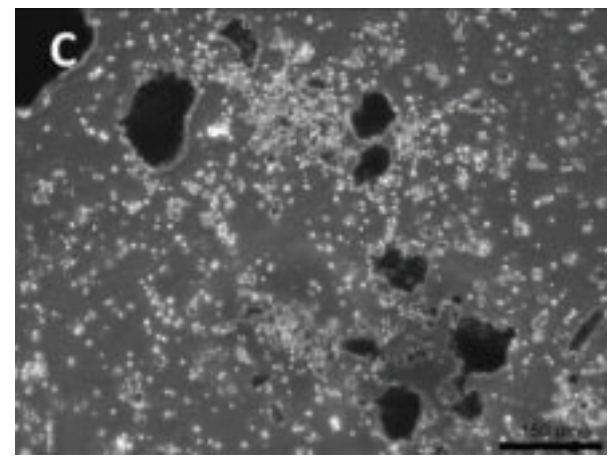

(c)

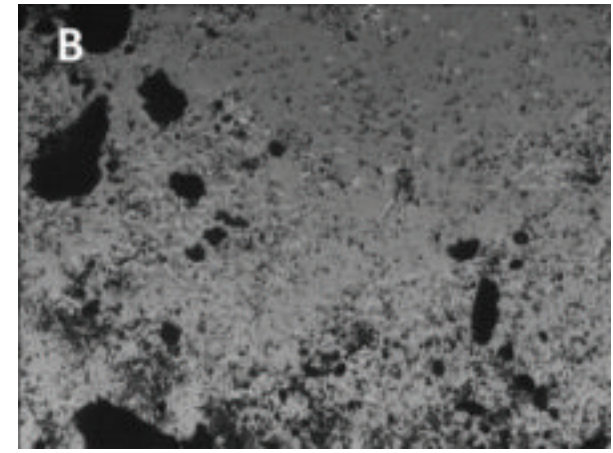

(b)

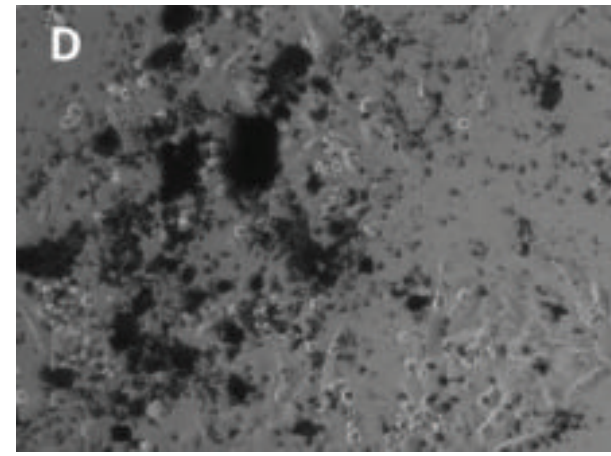

(d)

Figure 7: Images of the initial seeding of the scaffolds. (a) and (c) 2 hrs after seeding. (b) and (d) 24 hrs after seeding. (a) and (b) $\times 4$ magnification. (c) and (d) $\times 10$ magnification.

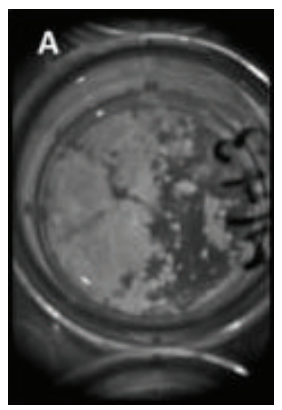

(a)

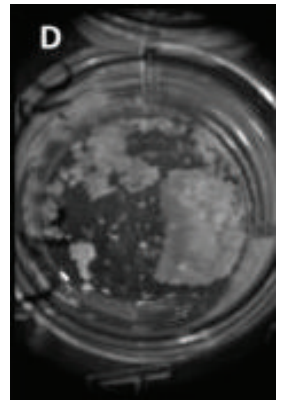

(d)

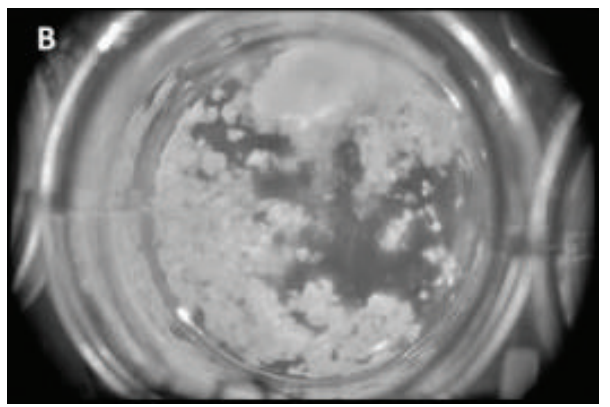

(b)

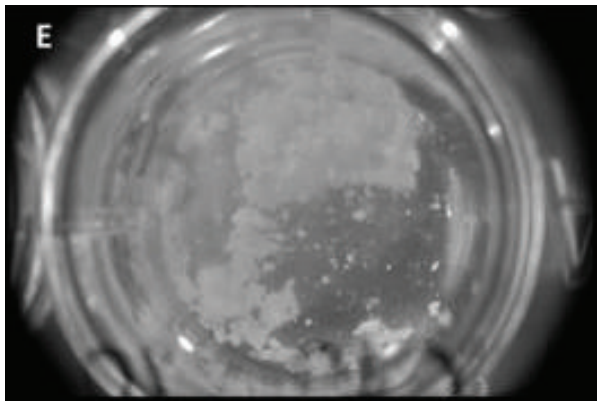

(e)

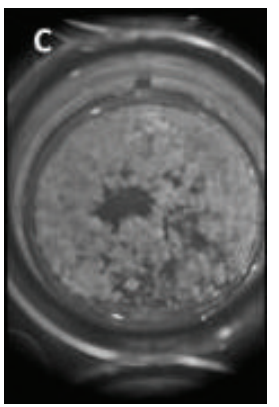

(c)

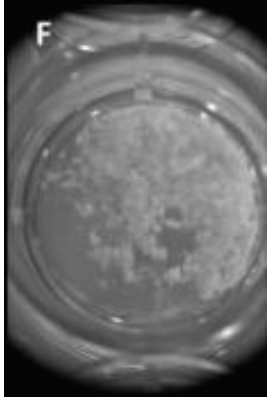

(f)

Figure 8: Scaffold morphology after seeding. (a) and (d) $48 \mathrm{hrs}$ after seeding. (b) and (e) $72 \mathrm{hrs}$ after seeding. (c) and (f) $192 \mathrm{hrs}$ after seeding ((a)-(c) $1 \mathrm{~mL}$ well volume; (d)-(f) $2 \mathrm{~mL}$ well volume). 


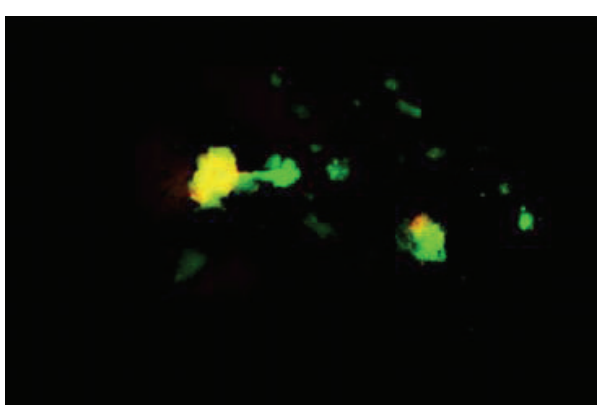

(a)

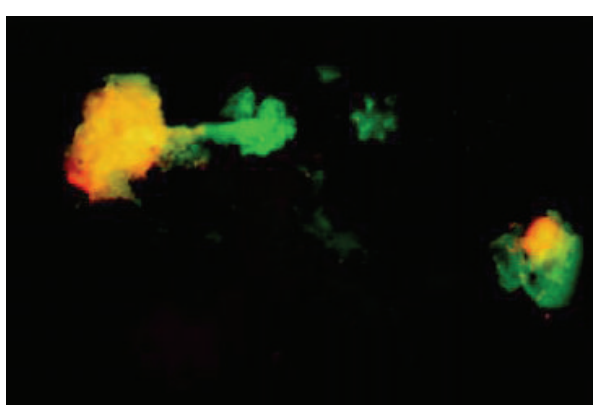

(b)

FIGURE 9: Live/Dead staining carried out on scaffold segments $192 \mathrm{hrs}$ after seeding. (a) 4x magnification. (b) 10x magnification.

the collagen isolation technique, type of collagen, and water content will affect the denaturation temperature [23]. Whilst DHT processing improves cross-link density, it also simultaneously leads to the denaturation of collagen molecules [24]. Along with this, the moisture content of the scaffold or the accumulation of solvent within it will change the denaturation temperature [16] and to overcome this problem we have utilized a lyophilization procedure prior to cross-linking.

Gamma irradiation (standard dose recommended by the European Pharmacopeia) was used for the sterilization of the collagen/ $\beta$-TCP scaffolds [25]. Although the formation of free radicals is not detected with electrospin resonance (ESR), gamma radiation is known to impede the mechanical properties of the material and speed the degradation process. To overcome this issue a DHT sterilization method, also described in literature [26], could be opted.

The swelling capacity of the scaffolds carries great importance for tissue culture in terms of cell growth and differentiation [27]. Whilst water absorption was seen in scaffolds subjected to 1 day of DHT processing, water absorption levels remained constant for scaffolds, which underwent longer DHT processing times. Swelling capacities depend on collagen and $\beta$-TCP content as well as cross-link density. A reported study has found a higher swelling ratio, possibly due to the utilization of only collagen matrix [21].

The scaffolds prepared in this study did not cause cytotoxic effects. A Live/Dead staining analysis found a high level of cell viability, implying that collagen/ $\beta$-TCP scaffolds remaining did not negatively affect cell survival. During in vitro disintegration tests, $\beta$-TCP particles disintegrated from the scaffolds. This could be attributed to the excessive crosslinking of the scaffold [28] or long DHT processing times [29]. This disintegration could give increased surface area and vascularization when implanted.

\section{Conclusion}

In this study, we have successfully developed porous collagen/ $\beta$-TCP scaffolds with an interconnected pore structure with $\beta$-TCP particles homogeneously distributed in the network of collagen fibrils. We have found that these scaffolds display complete biocompatibility, superior mechanical properties and can offer ease of handling. They are able to bend and be rolled, making it suitable for wide range of indications in terms of handling. The optimal DHT time was obtained by using data from characterization studies. Collectively, the findings from this study suggest that these cross-linked collagen/ $\beta$-TCP scaffolds fabricated via dehydrothermal processing show great potential for use in bone tissue engineering applications.

\section{Conflict of Interests}

The authors declare that there is no conflict of interests regarding the publication of this paper.

\section{Acknowledgments}

This project has been completed with the support of TUBITAK under the TUBITAK Project no. 7120905. The authors would like to thank BMT Calsis Health Technologies and the Bioengineering Research and Application Center, Hacettepe University, for their support. They would also like to thank Deniz Ozdil for proofreading this research paper.

\section{References}

[1] C. Wang, G. Meng, L. Zhang, Z. Xiong, and J. Liu, "Physical properties and biocompatibility of a core-sheath structure composite scaffold for bone tissue engineering in vitro," Journal of Biomedicine and Biotechnology, vol. 2012, Article ID 579141, 9 pages, 2012.

[2] M. Wagner-Ecker, P. Voltz, M. Egermann, and W. Richter, "The collagen component of biological bone graft substitutes promotes ectopic bone formation by human mesenchymal stem cells," Acta Biomaterialia, vol. 9, no. 7, pp. 7298-7307, 2013.

[3] T. Matsuno, T. Nakamura, K.-I. Kuremoto et al., "Development of $\beta$-tricalcium phosphate/collagen sponge composite for bone regeneration," Dental Materials Journal, vol. 25, no. 1, pp. 138$144,2006$.

[4] E. I. Oprita, L. Moldovan, O. Craciunescu, and O. Zarnescu, "In vitro behaviour of osteoblast cells seeded into a COL/ $\beta$-TCP composite scaffold," Central European Journal of Biology, vol. 3, no. 1, pp. 31-37, 2008.

[5] Y. Kang, S. Kim, J. Bishop, A. Khademhosseini, and Y. Yang, "The osteogenic differentiation of human bone marrow MSCs on HUVEC-derived ECM and $\beta$-TCP scaffold," Biomaterials, vol. 33, no. 29, pp. 6998-7007, 2012. 
[6] J. E. Maté-Sánchez de Val, P. Mazõn, J. L. C. Guirado et al., "Comparison of three hydroxyapatite/ $\beta$-tricalcium phosphate/ collagen ceramic scaffolds: an in vivo study," Journal of Biomedical Materials Research A, vol. 102, no. 4, pp. 1037-1046, 2014.

[7] T. Uemura, J. Dong, Y. Wang et al., "Transplantation of cultured bone cells using combinations of scaffolds and culture techniques," Biomaterials, vol. 24, no. 13, pp. 2277-2286, 2003.

[8] A. Bernstein, P. Niemeyer, G. Salzmann et al., "Microporous calcium phosphate ceramics as tissue engineering scaffolds for the repair of osteochondral defects: histological results," Acta Biomaterialia, vol. 9, no. 7, pp. 7490-7505, 2013.

[9] X. Zhang, M. Xu, X. Liu et al., "Restoration of critical-sized defects in the rabbit mandible using autologous bone marrow stromal cells hybridized with nano- $\beta$-tricalcium phosphate/ collagen scaffolds," Journal of Nanomaterials, vol. 2013, Article ID 913438, 8 pages, 2013.

[10] M. Ebrahimi, P. Pripatnanont, N. Monmaturapoj, and S. Suttapreyasri, "Fabrication and characterization of novel nano hydroxyapatite/beta-tricalcium phosphate scaffolds in three different composition ratios," Journal of Biomedical Materials Research Part A, vol. 100, no. 9, pp. 2260-2268, 2012.

[11] X.-F. Zhao, X.-D. Li, Y.-Q. Kang, and Q. Yuan, "Improved biocompatibility of novel poly(L-lactic acid)/ $\beta$-tricalcium phosphate scaffolds prepared by an organic solvent-free method," International Journal of Nanomedicine, vol. 6, pp. 1385-1390, 2011.

[12] H. J. Kim, U.-J. Kim, G. Vunjak-Novakovic, B.-H. Min, and D. L. Kaplan, "Influence of macroporous protein scaffolds on bone tissue engineering from bone marrow stem cells," Biomaterials, vol. 26, no. 21, pp. 4442-4452, 2005.

[13] E. I. Oprita, L. Moldovan, O. Craciunescu, W. Buzgariu, C. Tardei, and O. Zarnescu, "A bioactive collagen- $\beta$ tricalcium phosphate scaffold for tissue engineering," Central European Journal of Biology, vol. 1, no. 1, pp. 61-72, 2006.

[14] H. Nitzsche, A. Lochmann, H. Metz et al., "Fabrication and characterization of a biomimetic composite scaffold for bone defect repair," Journal of Biomedical Materials Research Part A, vol. 94, no. 1, pp. 298-307, 2010.

[15] N. Koushki, A. A. Katbab, H. Tavassoli, A. Jahanbakhsh, M. Majidi, and S. Bonakdar, "A new injectable biphasic hydrogel based on partially hydrolyzed polyacrylamide and nanohydroxyapatite as scaffold for osteochondral regeneration," RSC Advances, vol. 5, no. 12, pp. 9089-9096, 2015.

[16] M. G. Haugh, M. J. Jaasma, and F. J. O’Brien, “The effect of dehydrothermal treatment on the mechanical and structural properties of collagen-GAG scaffolds," Journal of Biomedical Materials Research-Part A, vol. 89, no. 2, pp. 363-369, 2009.

[17] A. Sionkowska and J. Kozłowska, "Properties and modification of porous 3-D collagen/hydroxyapatite composites," International Journal of Biological Macromolecules, vol. 52, no. 1, pp. 250-259, 2013.

[18] D. A. Wahl, E. Sachlos, C. Liu, and J. T. Czernuszka, "Controlling the processing of collagen-hydroxyapatite scaffolds for bone tissue engineering," Journal of Materials Science: Materials in Medicine, vol. 18, no. 2, pp. 201-209, 2007.

[19] J. Kozłowska and A. Sionkowska, "Effects of different crosslinking methods on the properties of collagen-calcium phosphate composite materials," International Journal of Biological Macromolecules, vol. 74, pp. 397-403, 2015.

[20] P. G. Coelho, M. E. Coimbra, C. Ribeiro et al., "Physico/chemical characterization and preliminary human histology assessment of a $\beta$-TCP particulate material for bone augmentation,"
Materials Science and Engineering C, vol. 29, no. 7, pp. 20852091, 2009.

[21] M. Safandowska and K. Pietrucha, "Effect of fish collagen modification on its thermal and rheological properties," International Journal of Biological Macromolecules, vol. 53, pp. 32-37, 2013.

[22] L. Salvatore, M. Madaghiele, C. Parisi, F. Gatti, and A. Sannino, "Crosslinking of micropatterned collagen-based nerve guides to modulate the expected half-life," Journal of Biomedical Materials Research A, vol. 102, no. 12, pp. 4406-4414, 2014.

[23] M. Madaghiele, A. Piccinno, M. Saponaro, A. Maffezzoli, and A. Sannino, "Collagen- and gelatine-based films sealing vascular prostheses: evaluation of the degree of crosslinking for optimal blood impermeability," Journal of Materials Science: Materials in Medicine, vol. 20, no. 10, pp. 1979-1989, 2009.

[24] H. Sugiura, S. Yunoki, E. Kondo, T. Ikoma, J. Tanaka, and K. Yasuda, "In vivo biological responses and bioresorption of tilapia scale collagen as a potential biomaterial," Journal of Biomaterials Science, Polymer Edition, vol. 20, no. 10, pp. 13531368, 2009.

[25] CPMP and CFPMP, The European Agency for the Evaluation of Medicinal Products Evaluation of Medicines for Human Use, European Agency for the Evaluation of Medicinal Products, London, UK, 2000.

[26] E. M. Noah, J. Chen, X. Jiao, I. Heschel, and N. Pallua, "Impact of sterilization on the porous design and cell behavior in collagen sponges prepared for tissue engineering," Biomaterials, vol. 23, no. 14, pp. 2855-2861, 2002.

[27] S.-N. Park, H. J. Lee, K. H. Lee, and H. Suh, "Biological characterization of EDC-crosslinked collagen-hyaluronic acid matrix in dermal tissue restoration," Biomaterials, vol. 24, no. 9, pp. 1631-1641, 2003.

[28] Y. Hu, L. Liu, W. Dan, N. Dan, Z. Gu, and X. Yu, "Synergistic effect of carbodiimide and dehydrothermal crosslinking on acellular dermal matrix," International Journal of Biological Macromolecules, vol. 55, pp. 221-230, 2013.

[29] M. Geiger and W. Friess, "Crosslinking of porous collagen carrier systems by dehydrothermal treatment," in Proceedings of the 3rd World Meeting APV/APGE, Berlin, Germany, 2000. 

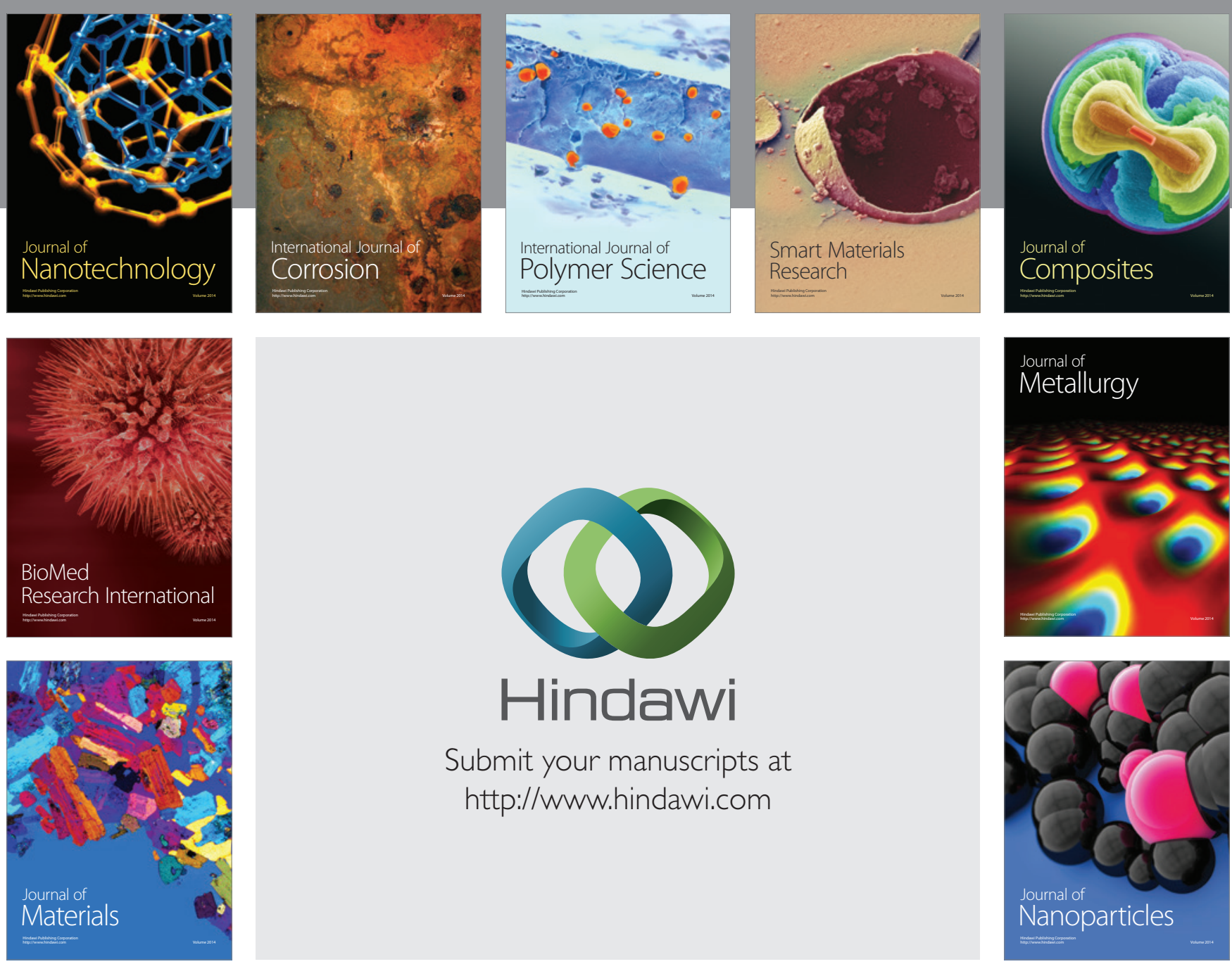

Submit your manuscripts at http://www.hindawi.com
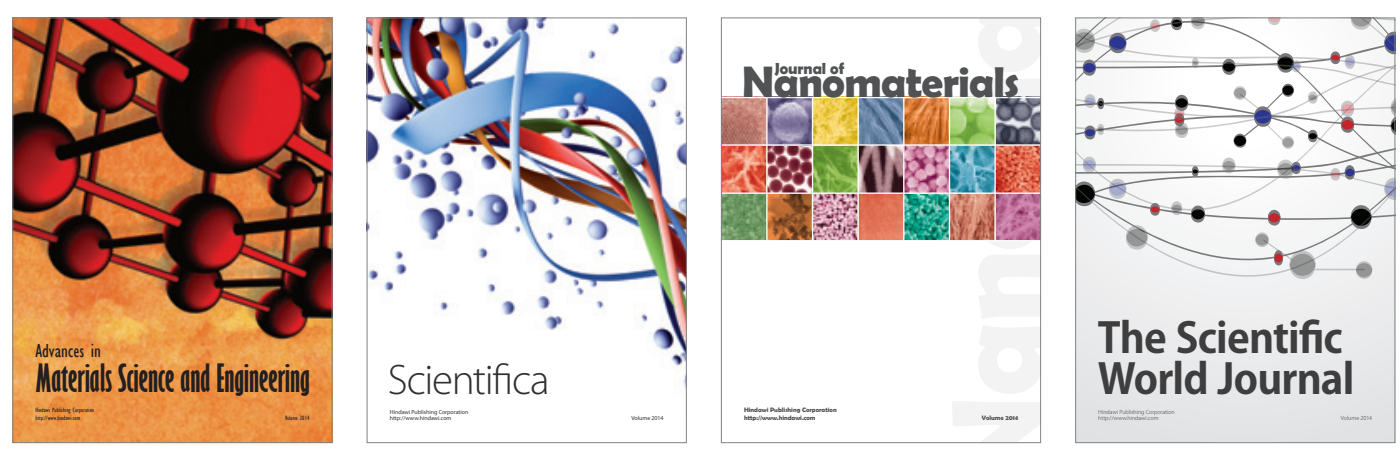

\section{The Scientific World Journal}
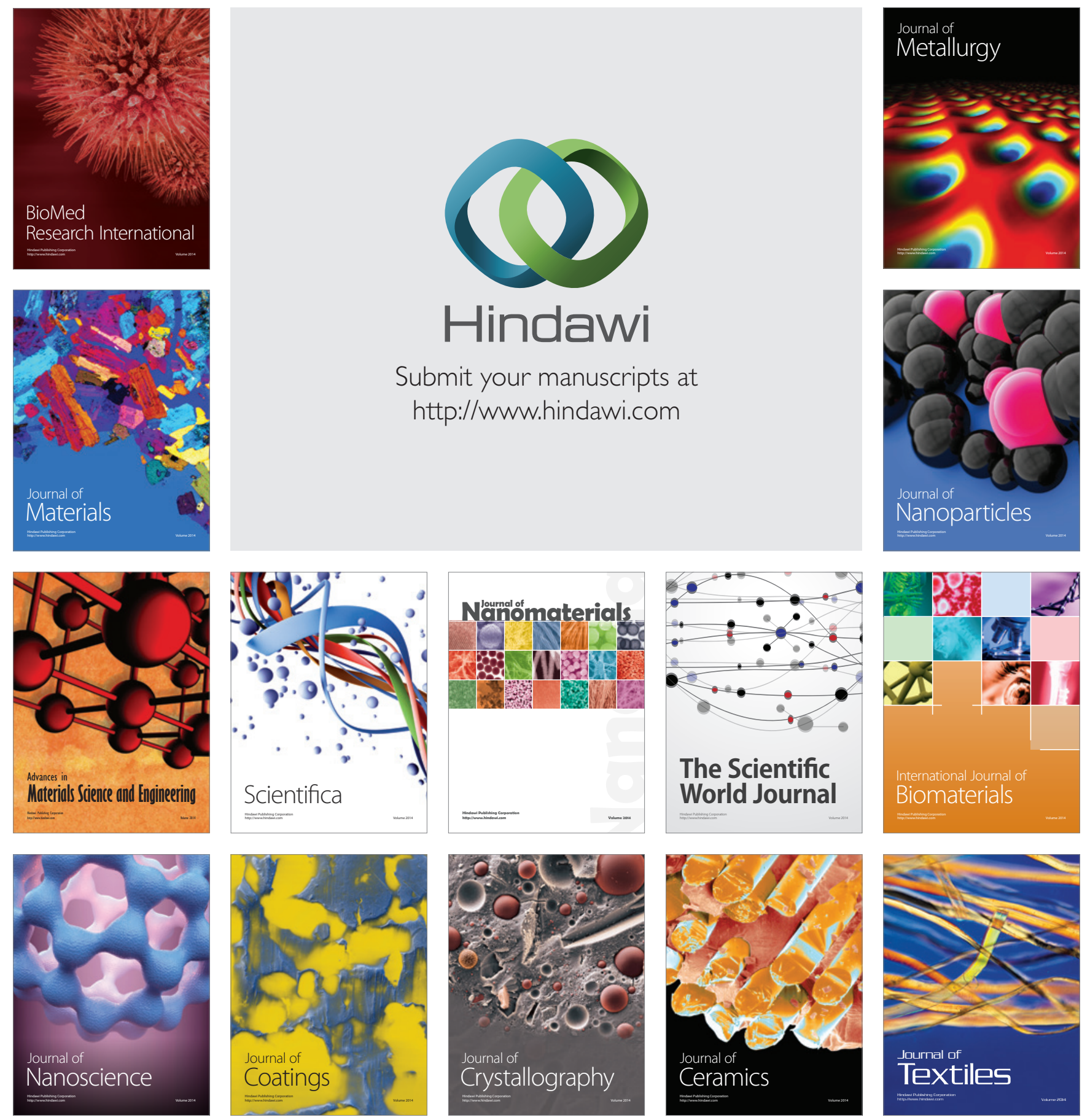